\title{
The Five Parameter Grain Boundary Character Distribution of $\alpha$-Ti Determined from Three-Dimensional Orientation Data
}

Madeleine N. Kelly, Krzysztof Glowinski, Noel T. Nuhfer, and Gregory S. Rohrer*

Department of Materials Science and Engineering, Carnegie Mellon University, Pittsburgh, Pennsylvania 15213, USA

Corresponding author: Gregory S. Rohrer, (412) 268-2696, rohrer@cmu.edu

Co-author email addresses: Madeleine N. Kelly (mnkelly@andrew.cmu.edu), Krzysztof Glowinski (kglowinski@ymail.com), Noel T. Nuhfer (tn06@andrew.cmu.edu)

\begin{abstract}
Commercially pure $\alpha$-Ti was serial sectioned using a Xe plasma focused ion beam (PFIB) scanning electron microscope and orientation maps were obtained on the parallel layers by electron backscatter diffraction. The orientations and shapes of 13900 grains and 92100 grain faces were characterized. The mean number of faces per grain was 14.2. The grain boundaries were classified according to the three misorientation parameters and two grain boundary orientation parameters. There were more grain boundaries with $180^{\circ}$-twist and $180^{\circ}$-tilt character than expected in a random distribution. Furthermore, grain boundary planes with prismatic orientations were more common than those with basal orientations. The grain boundary with the greatest relative area had a $28^{\circ} /[0001]$ misorientation and (31 $\left.\overline{4} 0\right)$ and $(7 \overline{2} \overline{5} 0)$ grain boundary planes. Compared to earlier instruments with Ga-ion sources, the milling speed of the PFIB makes it possible to collect ten times more data in a comparable time.
\end{abstract}

Keywords: grain boundaries, microstructure, titanium, grain boundary character distribution, three dimensional serial sectioning 


\section{Introduction}

In recent years, several techniques have been used to determine the three-dimensional (3D) microstructure of metallic and ceramic polycrystals. [1-6] These studies make it possible to measure the quantitative characteristics of microstructures that can only be estimated from twodimensional sections. In particular, they can be used to define all five of the crystallographic parameters of grain boundaries, which are known to influence materials properties such as corrosion resistance, [7] electrical resistance, [8] and strength. [9] The most common technique for 3D microstructure studies has been focused ion beam (FIB) serial sectioning in a scanning electron microscope (SEM). [10-15] In this experiment, the crystal orientations are mapped in a section plane, a Ga-ion beam is used to remove a thin layer, and the orientation mapping is repeated. A 3D orientation map can then be constructed from the resulting set of parallel layers.

The main limitation of the FIB SEM experiment is the time it takes to remove the material with the Ga-ion beam. Collecting a volume of data $1 \times 10^{5} \mu \mathrm{m}^{3}$ (a typical volume in past studies) requires several continuous days of automated data collection. Assuming a grain diameter of about $5 \mu \mathrm{m}$, this volume contains approximately $10^{3}$ grains and $7 \times 10^{3}$ grain faces. This is approximately the number of distinguishable grain boundary types in a cubic material, when the boundary parameters are resolved with an accuracy of $10^{\circ}$. [16] For hexagonal materials, the number of distinguishable grain boundaries is four times larger at the same angular resolution because of the lower symmetry. Therefore, to characterize a number of grain boundaries that meets or exceeds the number of distinguishable possibilities, it is necessary to sample larger volumes of material or more grains. For this reason, past 3D studies by FIB SEM have been limited to materials with cubic symmetry and relatively small grain sizes.

The recent availability of commercial Xe plasma focused ion beams (PFIBs) makes it possible to remove material at a faster rate and, in comparable times, characterize a much greater volume of material. [17] Compared to Ga FIBs, the PFIB has a greater current and the ion has a greater mass. As we show below, this instrument makes it possible to characterize a volume of at least $4.3 \times 10^{6} \mu \mathrm{m}^{3}$, which is more than ten times greater than the volume that has been characterized by the Ga FIB, in a similar amount of time. This paper has two main objectives. The first is to demonstrate the capabilities of the PFIB for developing 3D orientation maps. The second objective is to describe the five-parameter grain boundary character distribution of the hexagonal metal, $\alpha$-Ti. While many aspects of $\alpha$-Ti's microstructure, [18] texture, [19] and 
microtexture [20, 21] have been reported, the 3D structure and five-parameter grain boundary character distribution of $\alpha$-Ti (or any other hexagonal metal) has not been previously quantified.

\section{Experimental}

\subsection{Data Aquisition}

Commercially pure $\alpha$-Ti was prepared by first polishing the sample to a thickness of $80 \mu \mathrm{m}$. Pillars, approximately $300 \mu \mathrm{m}$ in width, were created by making incomplete, parallel cuts with a diamond saw. FEI's Helios ${ }^{\mathrm{TM}}$ PFIB Dual-Beam was used to collect the data. The FEI AutoSlice and View (EBS3 G2 1.6) software was used to control the milling and Oxford's HKL fast acquisition was used for orientation mapping on each slice. A schematic of the system is provided in Fig. 1, illustrating the geometry of the specimen and beams. The specimen was mounted onto a $36^{\circ}$ angle pre-tilted holder and the horizontal axes of the sample holder and stage were aligned (these axes are perpendicular to the page in Fig. 1). The vertical position was adjusted so that the region of interest was $4 \mathrm{~mm}$ from the pole piece. The stage was then tilted to $16^{\circ}$, which brings the region of interest to an inclination of $70^{\circ}$ with respect to the electron beam for electron backscatter diffraction (EBSD) pattern collection; this is referred to as the EBSD position (see Fig. 1(a)). For milling with the Xe-ion beam, the stage is rotated $180^{\circ}$ around the z-axis of the stage, so that the region of interest is parallel to the ion beam; this is referred to as the milling position (see Fig. 1(b)). The region of interest (ROI), which is perpendicular to the page in Figs. 1(a) and 1(b), is shown in an oblique projection of the sample in Fig. 1(c).

A platinum layer a few $\mathrm{nm}$ thick was deposited over the region of interest with the gas injection system needle at an accelerating voltage of $8 \mathrm{kV}$, beam current of $0.35 \mathrm{nA}, 80 \%$ overlap, and $250 \mu \mathrm{m}$ defocus. An X-shaped fiducial mark was then milled into the pillar at a position just below the region of interest. The Pt coating reduces charging and increases the contrast of the fiducial mark. The increased contrast is important because the AutoSlice and View software must recognize the fiducial mark to re-align's the pillar with each mill step so that the amount of material removed and the position of each EBSD scan is consistent. The throughthe-lens detector performed better than the Everhart-Thornley detector for imaging the fiducial mark. 
The total data set is made up of two volumes. The first is relatively small and the second is much larger. In both cases, the sample was ion milled at $30 \mathrm{kV}$ and $59 \mathrm{nA}$ using the PFIB. The EBSD data were collected using a $20 \mathrm{kV}$ and $5.5 \mathrm{nA}$ beam. A "rocking mill" with $5^{\circ}$ angle was used to minimize curtaining. In this procedure, the sample is milled at two different angles, +/$5^{\circ}$ about the rocking axis in Fig. 1(b). While the manufacturer's term, rocking, suggests continuous motion, the sample is actually fixed in one of the two positions during milling. The paths of the ions in the two positions are illustrated by the lines on the region of interest in Fig. 1(c). In the first, smaller volume, 55 parallel layers separated by $50 \mathrm{~nm}$ were characterized. The total area was $165 \mu \mathrm{m} \times 40 \mu \mathrm{m}$ and the EBSD step size was $0.5 \mu \mathrm{m}$. Operating at $24.9 \mathrm{~ms}$ per point, about 11 min were required to characterize each layer. The second, much larger data set is made up of 325 parallel layers, each separated by $100 \mathrm{~nm}$. The EBSD data was collected in an area of $218.5 \mu \mathrm{m}$ x $57.5 \mu \mathrm{m}$ with a step size of $0.5 \mu \mathrm{m}$. Operating at $24.9 \mathrm{~ms}$ per point, about 21 min were required to characterize each layer. The time required for milling was only $130 \mathrm{sec}$. For EBSD pattern collection, the camera was set to $4 \times 4$ pixel binning, an integration time of 4 ms, and eight frames were averaged for each point. For indexing, we selected a maximum of 32 reflectors. In the indexing software, we used band edge detection, standard divergence, a Hough resolution of 60 , and orientations were determined from between five and seven bands.

\subsection{Post Processing and Reconstruction}

Once the data were collected, the default Oxford 3D file type (CPR) was converted to the CTF file type using a Python script provided by Oxford instruments. This 3D CTF file was used for subsequent reconstruction and clean-up procedures in DREAM.3D. [22] The steps used in this process, known as a 'pipeline', were essentially the same as the pre-set reconstruction and clean-up pipelines provided in DREAM.3D, except for the parameters that were particular to this

dataset. Briefly, the stacks of 2D EBSD maps were aligned such that the overlap of pixels of similar orientations, belonging to adjacent slices and differing by no more than $5^{\circ}$, was maximized. The magnitudes of in-plane $x$ and $y$ shifts of the slices are illustrated in Fig. 2. In the current work, the in-plane shifts differed no more than 5 pixels as compared to much larger shifts in data collected by the Ga-ion FIB-SEM. The smaller shifts result from the new instrument's improved control of the stage position during slicing and the ability to carry out the sequential orientation mapping and milling without changing the stage tilt. 
The EBSD data were cleaned, first, by iteratively ascribing the parameters (orientations, image quality, etc.) of the most reliable neighbors to all voxels with undetermined orientations. Clusters of at least 50 voxels, whose orientations deviated from one another by less than $5^{\circ}$, were grouped into grains and average orientations were assigned to each grain. In addition, grains with fewer than four neighbors were removed. The reconstructed three-dimensional orientation maps, after clean-up, are shown in Fig. 3(a). Note that the orientations are colored with respect to the normal of the surface analyzed by EBSD. There was some texture in the sample; the [0001] poles were preferentially oriented $20^{\circ}$ to $40^{\circ}$ from the direction labeled ' $\mathrm{T}$ ' in Fig. 1(b), perpendicular to the sample's largest surface, with an intensity of 6 multiples of a random distribution (MRD). This texture is consistent with results in reference [20].

The surfaces of boundaries separating the grains were reconstructed in the form of meshes of planar triangular segments. The difference between the in-plane $(0.5 \mu \mathrm{m})$ and out-of-plane (0.1 $\mu \mathrm{m})$ spacing led to irregular triangle shapes. So, after aligning and cleaning the data from all slices, a DREAM.3D [22] filter was applied that changed the resolution of the larger (smaller) data set from $0.5 \mu \mathrm{m}$ and $0.1 \mu \mathrm{m}(0.5 \mu \mathrm{m}$ and $0.05 \mu \mathrm{m})$ to $0.5 \mu \mathrm{m}$ and $0.3 \mu \mathrm{m}(0.25 \mu \mathrm{m}$ and 0.15 $\mu \mathrm{m})$. Note that the resolution ratios for the two data sets were kept constant. In the future, it will clearly be more efficient to collect fewer slices with a more isotropic resolution. For each triangular segment, its normal, area, and misorientation between grains on its sides were determined. An example of one of these grains, and its triangular mesh, is illustrated in Fig. 3(b). Note that the triangles attached to triple lines are constrained in the smoothing procedure and they therefore preserve the stair-stepped characteristic of the voxelized data. The smaller data set contains $\sim 1500$ grains and $\sim 7800$ distinct grain faces (modeled by $\sim 1.6 \times 10^{6}$ triangular segments), while the larger one enclosed $\sim 12400$ grains and $\sim 84300$ grain faces (modeled by $\sim 1.5 \times 10^{7}$ triangular segments. For comparison, if the minimum number of neighbors is 2 , there are 12500 grains in the larger dataset. In other words, less than $1 \%$ of the grains have fewer than 4 sides. For a minimum grain size of 20 voxels and a minimum number of neighbors equal to 2 (4), we have 15400 (14900) grains in the larger dataset. Calculation of the GBCD using these different thresholds showed that there is almost no impact. This is because the GBCD is area weighted and these very small grains have little interfacial area.

\subsection{Computational Methods}


Although most Grain Boundary Character Distributions (GBCD) published so far were computed using a method based on dividing five-dimensional boundary space into bins and 'counting' boundaries ascribed to each bin, [23] in our analysis, we took advantage of an alternative approach that leads to more accurate distribution functions. [24] The idea is to estimate values of a distribution at given points by 'counting' boundaries that are close enough to those points; the closeness is measured in terms of metrics defined in the space of boundary parameters.

Let us recap the steps and basic assumptions of the new method. Mathematically, the boundary parameter space is a Cartesian product of the misorientation and boundary normal subspaces. Because the accuracies for determining grain misorientations and inclinations of boundary planes are significantly different, the calculation is simplified by using separate metric functions for each subspace. The distance in the misorientation subspace between two boundaries is given as $\min \left\{\operatorname{acos}\left[\left(\operatorname{tr} M^{\prime} M^{T}-1\right) / 2\right]\right\}$, where $M\left(M^{\prime}\right)$ denotes a misorientation matrix corresponding to the first (second) boundary, and the minimization is over all symmetrically equivalent representations of both boundaries. In the subspace of boundary normals, the distance is quantified as $\min \left[\sqrt{\left(\chi_{L}^{2}+\chi_{R}^{2}\right) / 2}\right]$, with $\chi_{i}=\arccos \mathbf{m}_{i} \cdot \mathbf{m}^{\prime}{ }_{i}$ and $\mathbf{m}_{i}$ $\left(\mathbf{m}_{i}^{\prime}\right)$ standing for the normals to the first (second) boundary expressed in the reference frames of its left $(L)$ and right $(R)$ grains, respectively.

GBCDs are typically presented as a series of sections for fixed misorientations and varying boundary normals. We followed this established convention and, to obtain a given section, the distribution was probed at points corresponding to the misorientation of interest and vectors pointing from the center towards a set of points evenly scattered on a unit hemisphere. For each sampling point, areas of mesh segments with misorientations closer than a limiting distance $\rho_{\mathrm{m}}$ to the fixed misorientation and having normals within another limiting distance $\rho_{\mathrm{p}}$ from the corresponding vector were summed. The obtained sums were normalized to express the distribution in conventional MRD units.

The radii $\rho_{\mathrm{m}}$ and $\rho_{\mathrm{p}}$ are selected to be consistent with the experimental resolution. The resolution of the grain boundary plane measurement has been determined to be approximately $7.5^{\circ}[23,24]$, so we use $\rho_{p}=7^{\circ}$. The threshold disorientation for the definition of a grain boundary in this work was $5^{\circ}$, so we selected a slightly larger value, $\rho_{\mathrm{m}}=6^{\circ}$. Angular ranges greater than the experimental resolution could be used for cases where the data is sparse, but that 
was not the case for these data. Here, limited tests using larger radii led to decreased maxima in the distributions, as more and more different types of grain boundaries are averaged together. If $n$ is the number of distinct grain boundaries (92100) and $v$ is the fractional volume of a subspace restricted by the radii $\rho_{\mathrm{m}}$ and $\rho_{\mathrm{p}}\left(1.21 \times 10^{-4}\right)$, then the error (one standard deviation $\sigma$ ) of the distribution value $f$ at a given point can be estimated as $\sigma=\sqrt{f /(n v)}$.

A projection of the five-dimensional GBCD onto the subspace of boundary planes is known as the Grain Boundary Plane Distribution (GBPD). The GBPD depicts the frequencies of occurrence of boundary planes averaged over all misorientations.

\section{Results}

The distribution of disorientation angles was computed from the combined data and is illustrated in Fig. 4. The distribution indicates that grain boundaries with disorientation angles smaller than $30^{\circ}$ occur more frequently than would be expected for random boundaries. To obtain more complete information concerning disorientations of adjacent grains, the disorientation axes in fixed intervals of the disorientations angle were analyzed (Fig. 5). They were obtained using the OIM Analysis software based on several two-dimensional EBSD maps taken from both data sets. For this purpose, we selected maps from different depths of the samples in such a way that no disorientation data were duplicated. In the distribution of disorientations, there is a peak of approximately 5 MRD for all boundaries with disorientations less than $30^{\circ}$; otherwise, the distribution is uniform.

Another relatively simple microstructural characteristic is the grain boundary plane distribution averaged over all misorientations. The GBPD obtained for the combined datasets is plotted in Fig. 6. This distribution indicates that, on average, boundaries having planes at the prismatic positions make up a greater fraction of the total grain boundary area than the basal planes. The corresponding relative areas are about 1.2 MRD and about 0.8 MRD, respectively. The range of the populations is relatively narrow meaning that the anisotropy of the boundary plane distribution is weak.

With knowledge of the disorientation distributions and the GBPD, we moved on to the analysis of the GBCD. In this analysis, we focused on the sections enclosing peaks with heights exceeding 1 MRD by more than $3 \sigma$, i.e., the sections for which we have the highest degree of confidence that the features are representative of the true anisotropy of the distribution. Most of 
such peaks occur in sections at misorientations corresponding to the over-represented disorientation angles in Fig. 4. Figures 7a-d present the GBCD sections for the misorientations with angles close to $20^{\circ}$ and axes corresponding to the vertices (7a-c) and the center (7d) of the standard stereographic triangle. For the $22^{\circ} /[0001]$ misorientation, peaks with heights of $7.9 \pm 0.8 \mathrm{MRD}$ are observed at the $(3 \overline{1} \overline{2} 0)$ pole and its symmetrically equivalent positions. These peaks correspond to boundaries which are simultaneously $180^{\circ}$-twist and $180^{\circ}$-tilt. [25] Note that $180^{\circ}$-twist boundaries are also referred to 'symmetric' boundaries. [26] Also, the maxima at the $(0 \overline{3} \overline{3} 1)$ plane in the $18^{\circ} /[10 \overline{1} 0]$ section and at the $(\overline{2} 4 \overline{2} 1)$ plane in the $21^{\circ} /$ [2$\overline{1} \overline{1} 0]$ section with the intensities of $4.7 \pm 0.6$ and $4.1 \pm 0.6 \mathrm{MRD}$, respectively, correspond to boundaries having the same special type of geometry. Besides the aforementioned peaks, it is observed - consistent with the GBPD - that boundaries with disorientations smaller than $30^{\circ}$ tend to have planes at the prismatic positions rather than at the basal locations. This tendency is also apparent for boundaries with the $20^{\circ} /[4 \overline{1} \overline{3} 2]$ misorientation (Fig. $\left.7 \mathrm{~d}\right)$. It is worth noting that the corresponding section does not exhibit any distinct peaks, but a ring-shaped maximum with values up to $4.3 \pm 0.6 \mathrm{MRD}$ is clearly seen. The same tendency is visible for boundaries with the $28^{\circ} /[0001]$ misorientation (Fig. 7e). However, in this case, local maxima can be distinguished at the $(31 \overline{4} 0)$ and $(7 \overline{2} \overline{5} 0)$ orientations, with relative areas of $8.5 \pm 0.9 \mathrm{MRD}$ and $7.9 \pm 0.8 \mathrm{MRD}$, respectively; again, the maxima correspond to $180^{\circ}$-twist and $180^{\circ}$-tilt boundaries. It should be noted that the latter section partially overlaps with the one for the $22^{\circ} /[0001]$ misorientation, however, it still reveals different peaks in the distribution. In the $90^{\circ} /[10 \overline{1} 0]$ section (Fig. 7f), there are two smeared maxima with heights about $5.5 \pm 0.7 \mathrm{MRD}$ centered at the $(\overline{1} 2 \overline{1} 3)$ and $(3, \overline{6}, 3,10)$ poles. These poles correspond to $180^{\circ}$-twist boundaries.

It has been shown that high populations of grain boundaries are correlated to low boundary energies. [27] The relationships between the disorientation angle and the energies of certain boundaries tilted about the [2$\overline{1} \overline{1} 0]$ and [10 $\overline{1} 0]$ axes have been computed by simulation for

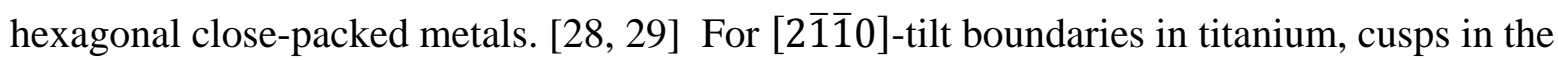
boundary energy were observed for the angles $31^{\circ}, 42^{\circ}, 61^{\circ}$, and $75^{\circ}$ and for [10 10$]$-tilt boundaries there were cusps at $28^{\circ}, 38^{\circ}, 58^{\circ}$, and $72^{\circ}$. In the section for the $31^{\circ} /[2 \overline{1} \overline{1} 0]$ misorientation (Fig. 7g), there is a peak near the (1013) pole corresponding to a boundary that is twist and $180^{\circ}$-tilt simultaneously; the height of that peak is $3.8 \pm 0.6 \mathrm{MRD}$. In the $75^{\circ} /[2 \overline{1} \overline{1} 0]$ section (Fig. 7h), there are several overlapping maxima with intensities up to $3.5 \pm 0.6 \mathrm{MRD}$ 
centered at the intersections of distinct zones of tilt boundaries. [25] For the other misorientations that are predicted to have low energies, the populations did not exceed $1 \mathrm{MRD}$ by at least $3 \sigma .[28,29]$

The highest intensities in the GBCD were for $180^{\circ}$-twist and $180^{\circ}$-tilt boundaries. Therefore, it was interesting to estimate the fractions of these two types of boundaries. For this purpose, the parameters $\alpha_{\mathrm{S}}$ and $\alpha_{\mathrm{I}}$, introduced in reference [26], were used. These parameters are approximate distances (in the boundary space) from a given boundary to the nearest $180^{\circ}$-twist and the nearest $180^{\circ}$-tilt boundaries, respectively. If $\alpha_{\mathrm{S}}$ or $\alpha_{\mathrm{I}}$ are less than a specified limit, then the boundary is classified adequately as near $-180^{\circ}$-twist or near- $180^{\circ}$-tilt. In previous work, the influence of the threshold values on the results was explored [26, 30]. Following that work [26], the limit of $8^{\circ}$ (embracing the accuracies of both misorientations and boundary normals) was assumed. With this limit, the fractions of near- $180^{\circ}$-twist and near- $180^{\circ}$-tilt boundaries among random boundaries are $0.7 \%$ and $11.3 \%$, respectively (for hexagonal symmetry). For titanium, they were $1.4 \%$ and $16.7 \%$, thus, it is clear that near- $180^{\circ}$-twist and near- $180^{\circ}$-tilt boundaries are overrepresented in this material.

For completeness, the twist and tilt characters of boundaries can be quantified using an analogous pair of parameters, $\alpha_{\mathrm{N}}$ and $\alpha_{\mathrm{L}}$, defined in reference [30]. It turned out that, using the same level of tolerance, the fraction of near-twist boundaries in titanium (11.5\%) is similar to that for random boundaries (11.3\%). In the case of tilt boundaries, it would be difficult to draw any conclusions from experimental data as $92.2 \%$ of random boundaries can be classified as near-tilt (within $8^{\circ}$ ).

Many microstructural characteristics can be estimated from 2D EBSD maps using stereological techniques. With 3D data, these characteristics can be obtained directly, avoiding a number of approximations. Those considered here are the grain size distribution (Fig. 8) and the distribution of the number of faces per grain (Fig. 9a). A histogram combining these two characteristics and presenting the fractions of grains with given numbers of faces and given grain diameters is shown in Fig. 9b. (To obtain graphs in Figs. 8 and 9, only the grains entirely contained in the microstructure images were taken into account, i.e., the grains cut by the outer surfaces of the images were neglected.) It can be seen that the number of faces per grain is correlated to the grain diameter (the Spearman's rank correlation coefficient is 0.89 ). 


\section{Discussion}

The number of distinguishable grain boundaries in a polycrystalline hexagonal material is four times larger than in a polycrystalline cubic material. If the five grain boundary parameters are discriminated with a resolution of $10^{\circ}$, then there are approximately $1.3 \times 10^{4}$ distinguishable grain boundaries in hexagonal materials. [16] If one wishes to measure the relative areas of different grain boundary types, at least this many boundaries must be observed. In the work, 9.2 $\mathrm{x} 10^{4}$ grain faces where characterized, which is more than seven times the number of distinguishable boundaries. It should be noted that the comparison of these numbers is not so simple. Each grain face is comprised of many triangular segments that span a range of boundary plane orientations (see Fig. 3(b)). While there is considerable degeneracy among the triangles that make up a grain face, they typically represent more than one grain boundary type. So, the number of grain boundaries characterized is greater the number of grain faces $\left(9.2 \times 10^{4}\right)$, but less than the number of triangular segments $\left(1.7 \times 10^{7}\right)$. Considering this, we can be confident that we observed many more grain boundaries than there are types of grain boundaries and should have an acceptable measure of the distribution, at least in the sections where the population is large.

The distribution of disorientation angles in $\alpha$-Ti has been measured previously [20] and is similar to the distribution shown in Fig. 4. However, the five-parameter GBCD is not known and, because of the large number of distinguishable boundaries, there are no previous 3D datasets for a hexagonal metal. The observed grain boundary plane distribution (see Fig. 5) for $\alpha-\mathrm{Ti}$ is relatively isotropic. In other words, the relative areas of different grain boundaries vary over a range that is smaller than observed in some cubic materials. For example, the GBPD varies from 0.8 MRD at the (0001) orientation to $1.2 \mathrm{MRD}$ at the prismatic orientations. The differences between the maximum and minimum in $\mathrm{Ni}$, [11] $\mathrm{SrTiO}_{3}$, [31] $\mathrm{MgO}$, [23] and WC [32] are 2.1 MRD, 0.8 MRD, 1.3 MRD, and 3.7 MRD, respectively. The difference for $\alpha$-Ti (0.4) is similar to that observed for Al. [33]

All GBCDs for hexagonal materials obtained before now were calculated using stereological approaches that partition the boundary space into discrete bins. [32, 21] Because the volume determined by $\rho_{\mathrm{m}}=6^{\circ}$ and $\rho_{\mathrm{p}}=7^{\circ}$ in the metric-based approach is larger compared to the volume of a typically used " $10^{\circ}$-bin" by a factor of 3.4 and because these volumes are a key factor in the normalization of the distributions, the intensities of peaks in the GBCDs obtained 
using the two methods cannot be directly compared. However, the shapes of the functions and the locations of the peaks can still be compared. For example, in the distribution of WC/WC boundaries in a WC-Co composite, [32] there was a strong peak for the (0001) planes in the section for the $30^{\circ} /[0001]$ misorientation. That peak was about four times higher compared to the maxima at the prismatic positions in that section. No such peak is observed for titanium (with $\rho_{\mathrm{m}}=6^{\circ}$ it would be seen in the $28^{\circ} /[0001]$ section presented Fig. 7(e)). For tungsten carbide, a sharp peak was also reported at the $(10 \overline{1} 0)$ pole for the $90^{\circ} /[10 \overline{1} 0]$ misorientation. This peak does not exist in the case of titanium (Fig. 7(f)). In the GBCDs computed for both WC [32] and Ti6Al4V alloy, [21] there were significant maxima for misorientations about the [2$\overline{1} \overline{1} 0]$ axis by large misorientation angles: $57^{\circ}, 85^{\circ}$, and $90^{\circ}$. For our samples, no reliable features of the distribution were visible for these misorientations. Summarizing, the GBCD obtained in this work is significantly different from GBCDs obtained in the previous studies of other hexagonal materials. Considering the significant differences between the materials being compared, this is not surprising. The Ti6Al4V alloy has a different composition and its microstructure was determined mainly by the $\beta$-to- $\alpha$ phase transformation during processing. WC has a very different structure and its near unity c-to-a ratio is one characteristic that leads to a high population of $90^{\circ} /[10 \overline{1} 0]$ grain boundaries.

There is considerable evidence that in materials with weak texture that evolve by grain growth, the GBCD is inversely related to the grain boundary energy. [34, 35] With knowledge of the GBCD, we can see the extent to which it correlates with the energies predicted by simulations. [28, 29] Similar comparisons between computed grain boundary energies and populations in face centered cubic metals showed moderate to strong correlations. [36, 37] The simulations computed the energies of certain tilt boundaries with misorientations about the [2 $\overline{1} \overline{1} 0]$ and [10 $\overline{1} 0]$ axes, configured to have the same plane on each side of the boundary. The populations of these same types of boundaries are presented in Fig. 10. The population of boundaries with all axes is also presented for reference. For all grain boundaries, there is an increase in population at small disorientation angles, which mimics the decrease in energy expected at small disorientation angle. However, this trend is not found for the specific tilt

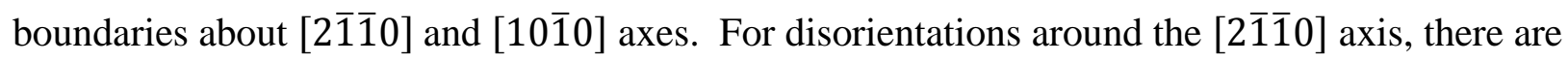
strong peaks in the population for the predicted low energy boundaries at $31^{\circ} /[2 \overline{1} \overline{1} 0]$ and $75^{\circ} /[2 \overline{1} \overline{1} 0]$, as we would expect. However, there is no indication of an increase in population 
at $42^{\circ} /[2 \overline{1} \overline{1} 0]$ and $61^{\circ} /[2 \overline{1} \overline{1} 0]$. For the population of grain boundaries with [10 $\left.\overline{1} 0\right]$ misorientations, there are weak local maxima in the population at the $38^{\circ} /[10 \overline{1} 0]$ and $58^{\circ} /[10 \overline{1} 0]$ cusps, but no signs of elevated populations at the $28^{\circ} /[10 \overline{1} 0]$ and $72^{\circ} /[10 \overline{1} 0]$ cusps. There is also a peak in population at $90^{\circ}$ for both types of misorientations, suggesting a low energy configuration, but the energy of this boundary was not simulated.

The results indicate that only some of the grain boundaries predicted to have low energies have elevated populations. One possible explanation is that the microstructure was formed at high temperature while the energies are computed at absolute zero. Furthermore, the predicted cusps for the [10 $\overline{1} 0]$ boundaries are weak; the minimum energy boundaries are only 15 to $20 \%$ lower than the energies of boundaries $5^{\circ}$ away, and these differences are likely to diminish at higher temperature where materials are processed. However, the cusps at $42^{\circ} /[2 \overline{1} \overline{1} 0]$ and $61^{\circ} /[2 \overline{1} \overline{1} 0]$ are much deeper and these boundaries did not have relatively larger populations. Finally, we note that the inverse correlation between energy and population is strongest in materials with random texture and this sample's texture is not random.

The mean number of faces per grain is 14.2. The grain with the most neighbors has 70 faces. However, one should note that both the mean and maximum are sensitive to the clean-up procedure and change when different thresholds are selected for the minimum grain size. The results are reported for a grain size threshold of 50 voxels. This threshold is selected to be large enough for the grain shape to be approximated by the voxelized data, but small enough so that large numbers of grains are not eliminated. To illustrate the sensitivity of topological parameters to this choice, other thresholds were selected; if the threshold is taken to be 30 (20) voxels, the mean number of faces was 13.8 (13.4) and the maximum number of faces was 75 (81). Note that the range of the mean number of faces for these different choices spans the number determined by Rowenhorst et al. [3] for $\beta$-Ti, 13.7. The similarity of the average numbers of grain faces in hexagonal and cubic Ti suggests that crystal symmetry does not strongly influence the topology of grains in a dense compact. The clean-up procedures also influence the average grain diameter, which can vary from 2.8 to $3.2 \mu \mathrm{m}$, depending on the assumption of the minimum grain size. The correlation between grain size and the number of faces reported here (see Fig. 9) is similar to the relationship observed for $\beta$-brass. [38]

The ability to characterize enough grain boundaries to determine the GBCD for a hexagonal material was enabled by the Xe PFIB, which is able to remove material faster than conventional 
Ga-ion FIBs. The Xe ion plasma source can be generated at a current of up to $1.3 \mu \mathrm{A}$ whereas the Ga ion source can only produce a current up to $50 \mathrm{nA}$. It should be noted however, that in neither case are the highest currents used, because they will damage the surface and degrade the diffraction patterns. However, because of the Xe plasma beam's characteristics, which include the ability to defocus during milling, and the rocking of the sample to decrease curtaining, samples can be milled at currents approximately ten times greater and still produce surfaces suitable for EBSD. The higher current of the Xe PFIB allows for much faster milling rates which allows for the acquisition of much larger data sets. Some of the materials that have been studied by Ga-ion FIB for 3D orientation analysis are $\mathrm{Y}_{2} \mathrm{O}_{3}$, [10] Ferrite, [14] and Austenite. [15] The volumes of these materials are: $30 \mu \mathrm{m}$ x $40 \mu \mathrm{m}$ x $17 \mu \mathrm{m}\left(\mathrm{Y}_{2} \mathrm{O}_{3}\right), 40 \mu \mathrm{m}$ x $35 \mu \mathrm{m}$ x $35 \mu \mathrm{m}$ (Ferrite), $65 \mu \mathrm{m}$ x $40 \mu \mathrm{m}$ x $20 \mu \mathrm{m}$ (Austenite). The volume of material analyzed in the present work was approximately 10 times greater. It should be noted that the rate-limiting step in the measurement of 3D orientation maps is now the EBSD measurement, not the milling. With the PFIB system used here, the milling time is roughly $10 \%$ of the time needed to measure the orientations. To our knowledge, this is the largest collection of grains $\left(1.4 \times 10^{4}\right)$ to be measured in $3 \mathrm{D}$ by a FIB. In fact, the only data set we are aware of that contains more grains $\left(1.9 \times 10^{4}\right)$ was obtained by manual serial sectioning. [39] Larger volume 3D reconstructions with orientation information can give us more statistically significant grain boundary character distributions, especially for lower symmetry materials and thus we will be able to analyze more types of materials than was possible before.

\section{Conclusions}

The grain boundary character distribution of $\alpha$-Ti has been determined from 3D EBSD data. Prismatic planes make up relatively more grain boundary area than basal planes, but overall, the anisotropy of the distribution is relatively weak compared to other materials. $180^{\circ}$-twist and $180^{\circ}$-tilt boundaries are significantly over-represented when compared to the random distribution. These types of boundaries dominate at the most highly populated misorientations, such as $28^{\circ} /[0001]$. Other features of the microstructure, including the average number of faces per grain and the dependence of the number of grain faces on the grain diameter are similar to the characteristics previously reported for cubic metals. The PFIB's increased milling rate, 
which made it possible to characterize more then 92,000 grain faces, greatly enhances our ability to characterize microstructures.

\section{Acknowledgement}

K.G. received funds for his work from the National Science Center Poland based on DEC2014/12/T/ST8/00086. G.S.R. acknowledges support from the National Science Foundation under MRI grant DMR 1428480. M.N.K. acknowledges support from the ONR-MURI program (grant no. N00014-11-0678). The authors acknowledge use of the Materials Characterization Facility at Carnegie Mellon University supported by grant MCF-677785. 


\section{Figure Captions}

Figure 1. Schematic of the Xe-ion PFIB. (a) In the EBSD position, the region of interest (ROI) makes a $70^{\circ}$ angle with the electron beam. (b) In the milling position, the ROI is parallel to the Xe-ion beam. The change from the EBSD position to the milling position is accomplished by a $180^{\circ}$ rotation around the z-axis. (c) In oblique projection, the ROI is visible and the paths of the ions in the two different positions are shown by the dashed lines.

(a) In the milling position, the combined $16^{\circ}$ stage tilt and $36^{\circ}$ inclination of the sample holder bring the surface of interest parallel to the Xe ion beam. (b) When the sample is rotated $180^{\circ}$ around the $\mathrm{z}$-axis of the microscope stage, it makes a $70^{\circ}$ angle with the electron beam, and this is the EBSD position. The direction $\mathrm{T}$ is the axis of preferred orientation.

Figure 2. Scatter plot illustrating $x$ and $y$ shifts necessary to align subsequent slices in 3D EBSD data collected from various materials. Shifts for $\mathrm{Ti}$ data, indicated as $\mathrm{Ti}_{\text {big }}$ and $\mathrm{Ti}_{\text {small, }}$ acquired in this work utilizing Xe - Plasma FIB-SEM are compared to those that were needed to align the yttria, [10] ferrite, [14] pure nickel, [11] and superalloy IN100 [12] data obtained in earlier works using Ga ion FIB-SEM.

Figure 3. (a) Three-dimensional orientation maps (after clean-up and reconstruction) of the smaller (top) and the larger (bottom) titanium sample volumes. Maps are colored according to the inverse pole figure (IPF). (b) Example of one of the larger grains and the mesh modeling the boundaries. Triangles are colored according to their normal using the same IPF coloring scheme as in (a).

Figure 4. Distribution of disorientation angles for $\alpha$-Ti (bars) and random grain boundaries (line).

Figure 5. Scatter plots illustrating the disorientation axes in fixed intervals of the disorientations angle for $\alpha$-Ti. In each plot are the axes (in the equal-area projection) corresponding to a range of disorientation angles centered at a given value $\pm 5^{\circ}$. Only large-angle boundaries $\left(>15^{\circ}\right)$ are taken into consideration. 
Figure 6. Distribution of boundary planes (GBPD) for all misorientations displayed in the stereographic projection. Units are multiples of the random distribution.

Figure 7. Sections for selected misorientations through the Grain Boundary Character Distribution of $\alpha$-Ti. In each pair, the upper plot depicts populations of boundaries as a function of boundary plane orientation, while the lower one is the corresponding error. Contours are plotted in stereographic projections and given in multiples of the random distribution.

Figure 8. Grain size distribution obtained for the larger Ti sample. The domain is limited to 8 $\mu \mathrm{m}$ to emphasize the most common grains. All grain sizes are displayed in Fig. 9(b).

Figure 9. (a) Distribution of numbers of faces per grain obtained for the larger Ti sample. The domain is limited at 40 faces to emphasize the most common grains. (b) Fractions of grains as a function of grain diameters (abscissa) and numbers of grain faces (ordinate) in the same sample. Dimensions of bins are $0.25 \mu \mathrm{m} \times 2 \mu \mathrm{m}$.

Figure 10. Population of grain boundaries for disorientations about the [2 $\overline{1} \overline{1} 0]$ and [10 $\overline{1} 0]$ axes and for all axes. For the misorientations around the [2 $\overline{1} \overline{1} 0]$ and [10 $\overline{1} 0]$, the population is the average of all boundaries within $5^{\circ}$ of the misorientation and $14^{\circ}$ of the appropriate grain boundary plane, which has the same orientation on both sides of the boundary. 


\section{Reference Cited}

[1] D.M. Saylor, A. Morawiec, G.S. Rohrer. Distribution and energies of grain boundaries in magnesia as a function of five degrees of freedom, Journal of the American Ceramic Society 85 (2002) 3081-3083. http://dx.doi.org/ 10.1111/j.1151-2916.2002.tb00583.x

[2] M.A. Groeber, B.K. Haley, M.D. Uchic, D.M. Dimiduk, S. Ghosh. 3D reconstruction and characterization of polycrystalline microstructures using a FIB-SEM system, Materials Characterization 57 (2006) 259-273. http://dx.doi.org/10.1016/j.matchar.2006.01.019

[3] D.J. Rowenhorst, A.C. Lewis, G. Spanos. Three-dimensional analysis of grain topology and interface curvature in a beta-titanium alloy, Acta Materialia 58 (2010) 5511-5519. http://dx.doi.org/10.1016/j.actamat.2010.06.030

[4] M.P. Echlin, A. Mottura, C.J. Torbet, T.M. Pollock. A new TriBeam system for threedimensional multimodal materials analysis, Review of Scientific Instruments 83 (2012) 023701. http://dx.doi.org/10.1063/1.3680111

[5] C.M. Hefferan, J. Lind, S.F. Li, U. Lienert, A.D. Rollett, R.M. Suter. Observation of recovery and recrystallization in high-purity aluminum measured with forward modeling analysis of high-energy diffraction microscopy, Acta Materialia 60 (2012) 4311-4318. http://dx.doi.org/10.1016/j.actamat.2012.04.020

[6] M. Syha, W. Rheinheimer, M. Baurer, E.M. Lauridsen, W. Ludwig, D. Weygand, P. Gumbsch. Three-dimensional grain structure of sintered bulk strontium titanate from X-ray diffraction contrast tomography, Scripta Materialia 66 (2012) 1-4.

http://dx.doi.org/10.1016/j.scriptamat.2011.08.005

[7] T. Watanabe. Grain boundary engineering: historical perspective and future prospects, Journal of Materials Science 46 (2011) 4095-4115. http://dx.doi.org/10.1007/s10853-011-5393-z [8] T. Sun, B. Yao, A.P. Warren, K. Barmak, M.F. Toney, R.E. Peale, K.R. Coffey. Surface and grain-boundary scattering in nanometric Cu films, Physical Review B 81 (2010) 155454. http://dx.doi.org/10.1103/PhysRevB.81.155454

[9] C.S. Kim, A.D. Rollett, G.S. Rohrer. Grain boundary planes: New dimensions in the grain boundary character distribution, Scripta Materialia 54 (2006) 1005-1009.

http://dx.doi.org/10.1016/j.scriptamat.2005.11.071

[10] S.J. Dillon, G.S. Rohrer. Characterization of the Grain-Boundary Character and Energy Distributions of Yttria Using Automated Serial Sectioning and EBSD in the FIB, Journal of the American Ceramic Society 92 (2009) 1580-1585. http://dx.doi.org/10.1111/j.15512916.2009.03064.x

[11] J. Li, S.J. Dillon, G.S. Rohrer. Relative grain boundary area and energy distributions in nickel, Acta Materialia 57 (2009) 4304-4311. http://dx.doi.org/10.1016/j.actamat.2009.06.004 [12] G.S. Rohrer, J. Li, S. Lee, A.D. Rollett, M. Groeber, M.D. Uchic. Deriving grain boundary character distributions and relative grain boundary energies from three-dimensional EBSD data, Materials Science and Technology 26 (2010) 661-669. http://dx.doi.org/10.1179/026708309x12468927349370

[13] S.J. Dillon, L. Helmick, H.M. Miller, L. Wilson, R. Gemman, R.V. Petrova, K. Barmak, G.S. Rohrer, P.A. Salvador. The Orientation Distributions of Lines, Surfaces, and Interfaces around Three-Phase Boundaries in Solid Oxide Fuel Cell Cathodes, Journal of the American Ceramic Society 94 (2011) 4045-4051. http://dx.doi.org/10.1111/j.1551-2916.2011.04673.x [14] H. Beladi, G.S. Rohrer. The relative grain boundary area and energy distributions in a ferritic steel determined from three-dimensional electron backscatter diffraction maps, Acta Materialia 61 (2013) 1404-1412. http://dx.doi.org/10.1016/j.actamat.2012.11.017 
[15] H. Beladi, N.T. Nuhfer, G.S. Rohrer. The five-parameter grain boundary character and energy distributions of a fully austenitic high-manganese steel using three dimensional data, Acta Materialia 70 (2014) 281-289. http://dx.doi.org/10.1016/j.actamat.2014.02.038

[16] G. Rohrer, D. Saylor, B. El Dasher, B. Adams, A. Rollett, P. Wynblatt. The distribution of internal interfaces in polycrystals, Zeitschrift Fur Metallkunde 95 (2004) 197-214.

[17] T.L. Burnett, R. Kelley, B. Winiarski, L. Contreras, M. Daly, A. Gholinia, M.G. Burke, P.J. Withers. Large volume serial section tomography by Xe Plasma FIB dual beam microscopy, Ultramicroscopy 161 (2016) 119-129. http://dx.doi.org/10.1016/j.ultramic.2015.11.001

[18] I. Weiss, S.L. Semiatin. Thermomechanical processing of alpha titanium alloys - an overview, Materials Science and Engineering a-Structural Materials Properties Microstructure and Processing 263 (1999) 243-256. http://dx.doi.org/10.1016/s0921-5093(98)01155-1

[19] F. Wagner, N. Bozzolo, O. Van Landuyt, T. Grosdidier. Evolution of recrystallisation texture and microstructure in low alloyed titanium sheets, Acta Materialia 50 (2002) 1245-1259. http://dx.doi.org/10.1016/s1359-6454(01)00427-x

[20] Y. Hu, V. Randle. An electron backscatter diffraction analysis of misorientation distributions in titanium alloys, Scripta Materialia 56 (2007) 1051-1054.

http://dx.doi.org/10.1016/j.scriptamat.2007.02.030

[21] V. Randle, G.S. Rohrer, Y. Hu. Five-parameter grain boundary analysis of a titanium alloy before and after low-temperature annealing, Scripta Materialia 58 (2008) 183-186. http://dx.doi.org/10.1016/j.scriptamat.2007.09.044

[22] M.A. Groeber, M.A. Jackson. DREAM.3D: A Digital Representation Environment for the Analysis of, Microstructure in 3D, Integrating Materials and Manufacturing Innovation 3 (2014) 5.

[23] D.M. Saylor, A. Morawiec, G.S. Rohrer. Distribution of grain boundaries in magnesia as a function of five macroscopic parameters, Acta Materialia 51 (2003) 3663-3674.

http://dx.doi.org/10.1016/s1359-6454(03)00181-2

[24] K. Glowinski, A. Morawiec. Analysis of Experimental Grain Boundary Distributions Based on Boundary-Space Metrics, Metallurgical and Materials Transactions A-Physical Metallurgy and Materials Science 45A (2014) 3189-3194. http://dx.doi.org/10.1007/s11661-0142325-y

[25] K. Glowinski, A. Morawiec. Twist, tilt, and symmetric grain boundaries in hexagonal materials, Journal of Materials Science 49 (2014) 3936-3942. http://dx.doi.org/10.1007/s10853013-7958-5

[26] K. Glowinski. On identification of symmetric and improperly quasi-symmetric grain boundaries, Journal of Applied Crystallography 47 (2014) 726-731.

http://dx.doi.org/10.1107/s160057671400435x

[27] G.S. Rohrer. Grain boundary energy anisotropy: a review, Journal of Materials Science 46 (2011) 5881-5895. http://dx.doi.org/10.1007/s10853-011-5677-3

[28] J. Wang, I.J. Beyerlein. Atomic structures of symmetric tilt grain boundaries in hexagonal close packed (hcp) crystals, Modelling and Simulation in Materials Science and Engineering 20 (2012) 024002. http://dx.doi.org/10.1088/0965-0393/20/2/024002

[29] J. Wang, I.J. Beyerlein. Atomic Structures of Symmetric Tilt Grain Boundaries in Hexagonal Close-Packed (hcp) Crystals, Metallurgical and Materials Transactions A-Physical Metallurgy and Materials Science 43A (2012) 3556-3569. http://dx.doi.org/10.1007/s11661-012$1177-6$ 
[30] A. Morawiec, K. Glowinski. On "macroscopic" characterization of mixed grain boundaries, Acta Materialia 61 (2013) 5756-5767.

http://dx.doi.org/10.1016/j.actamat.2013.06.019

[31] D.M. Saylor, B. El Dasher, T. Sano, G.S. Rohrer. Distribution of grain boundaries in

$\mathrm{SrTiO3}$ as a function of five macroscopic parameters, Journal of the American Ceramic Society 87 (2004) 670-676.

[32] C.S. Kim, T.R. Massa, G.S. Rohrer. Interface character distributions in WC-Co composites, Journal of the American Ceramic Society 91 (2008) 996-1001. http://dx.doi.org/10.1111/j.1551-2916.2007.02226.x

[33] D.M. Saylor, B.S. El Dasher, A.D. Rollett, G.S. Rohrer. Distribution of grain boundaries in aluminum as a function of five macroscopic parameters, Acta Materialia 52 (2004) 36493655. http://dx.doi.org/10.1016/j.actamat.2004.04.018

[34] S.J. Dillon, G.S. Rohrer. Mechanism for the development of anisotropic grain boundary character distributions during normal grain growth, Acta Materialia 57 (2009) 1-7. http://dx.doi.org/10.1016/j.actamat.2008.08.062

[35] G.S. Rohrer. Measuring and Interpreting the Structure of Grain-Boundary Networks, Journal of the American Ceramic Society 94 (2011) 633-646. http://dx.doi.org/10.1111/j.15512916.2011.04384.x

[36] G.S. Rohrer, E.A. Holm, A.D. Rollett, S.M. Foiles, J. Li, D.L. Olmsted. Comparing calculated and measured grain boundary energies in nickel, Acta Materialia 58 (2010) 50635069. http://dx.doi.org/10.1016/j.actamat.2010.05.042

[37] E.A. Holm, G.S. Rohrer, S.M. Foiles, A.D. Rollett, H.M. Miller, D.L. Olmsted. Validating computed grain boundary energies in fcc metals using the grain boundary character distribution, Acta Materialia 59 (2011) 5250-5256.

http://dx.doi.org/10.1016/j.actamat.2011.05.001

[38] F.C. Hull. Plane Section and Spatial Characteristics of Equiaxed beta-brass Grains, Materials Science and Technology 4 (1988) 778-785.

[39] S.E. Dillard, J.F. Bingert, D. Thoma, B. Hamann. Construction of simplified boundary surfaces from serial-sectioned metal micrographs, Ieee Transactions on Visualization and Computer Graphics 13 (2007) 1528-1535. http://dx.doi.org/10.1109/tvcg.2007.70543 


\section{Figure 1}

(a)

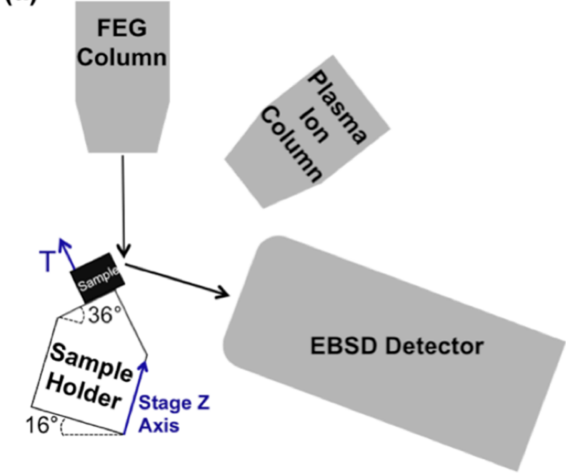

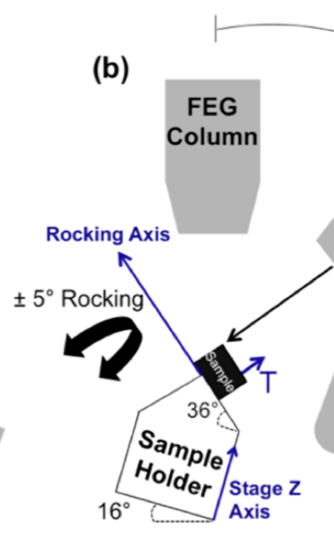

$52^{\circ}$

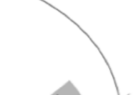

(c)

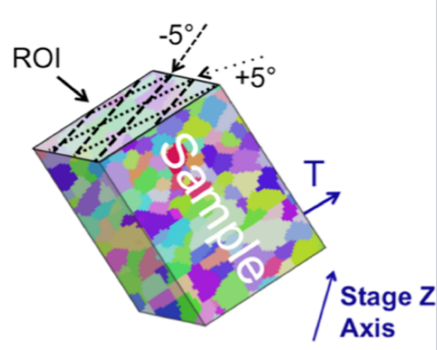

EBSD Detector 


\section{Figure 2}

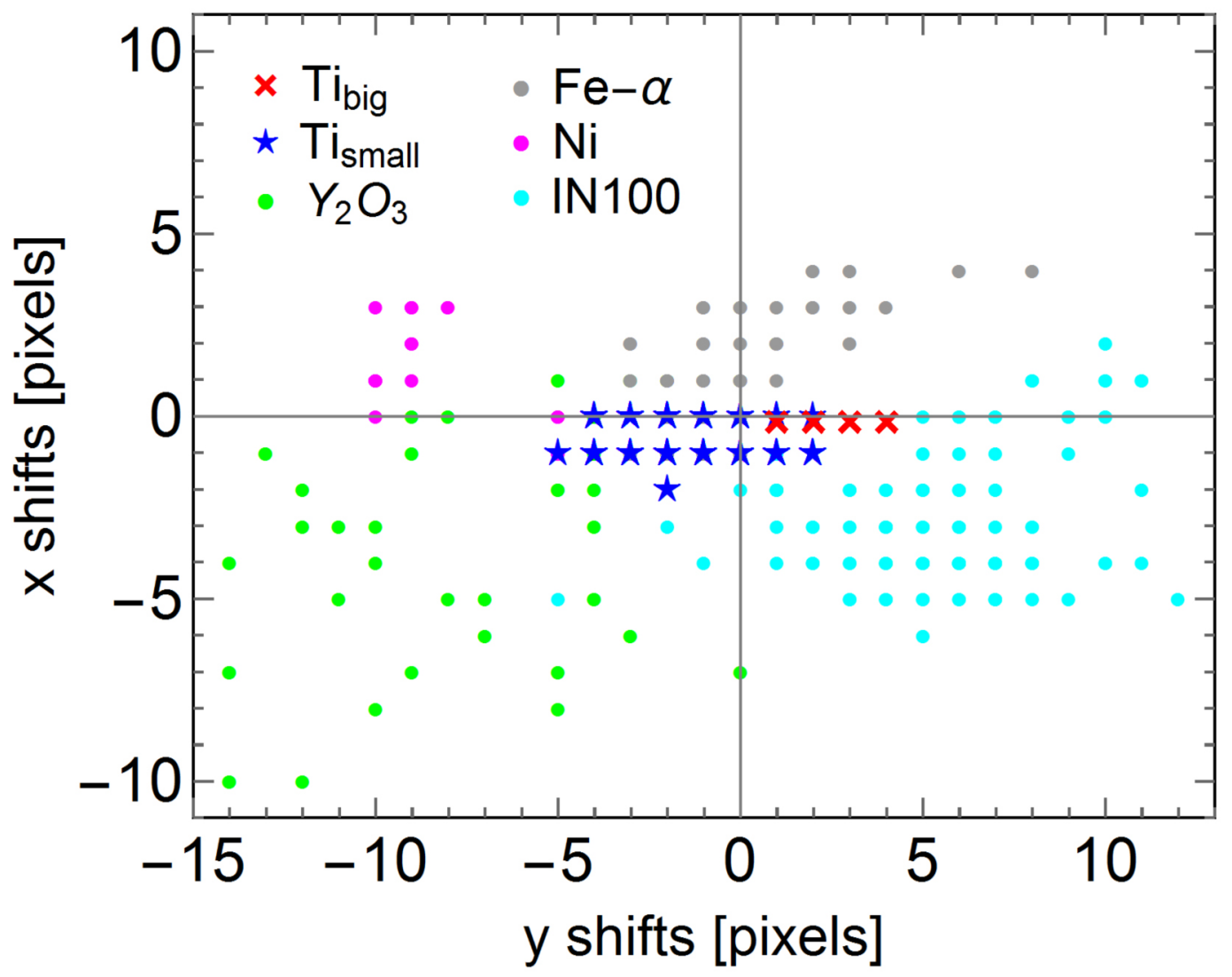




\section{Figure 3}

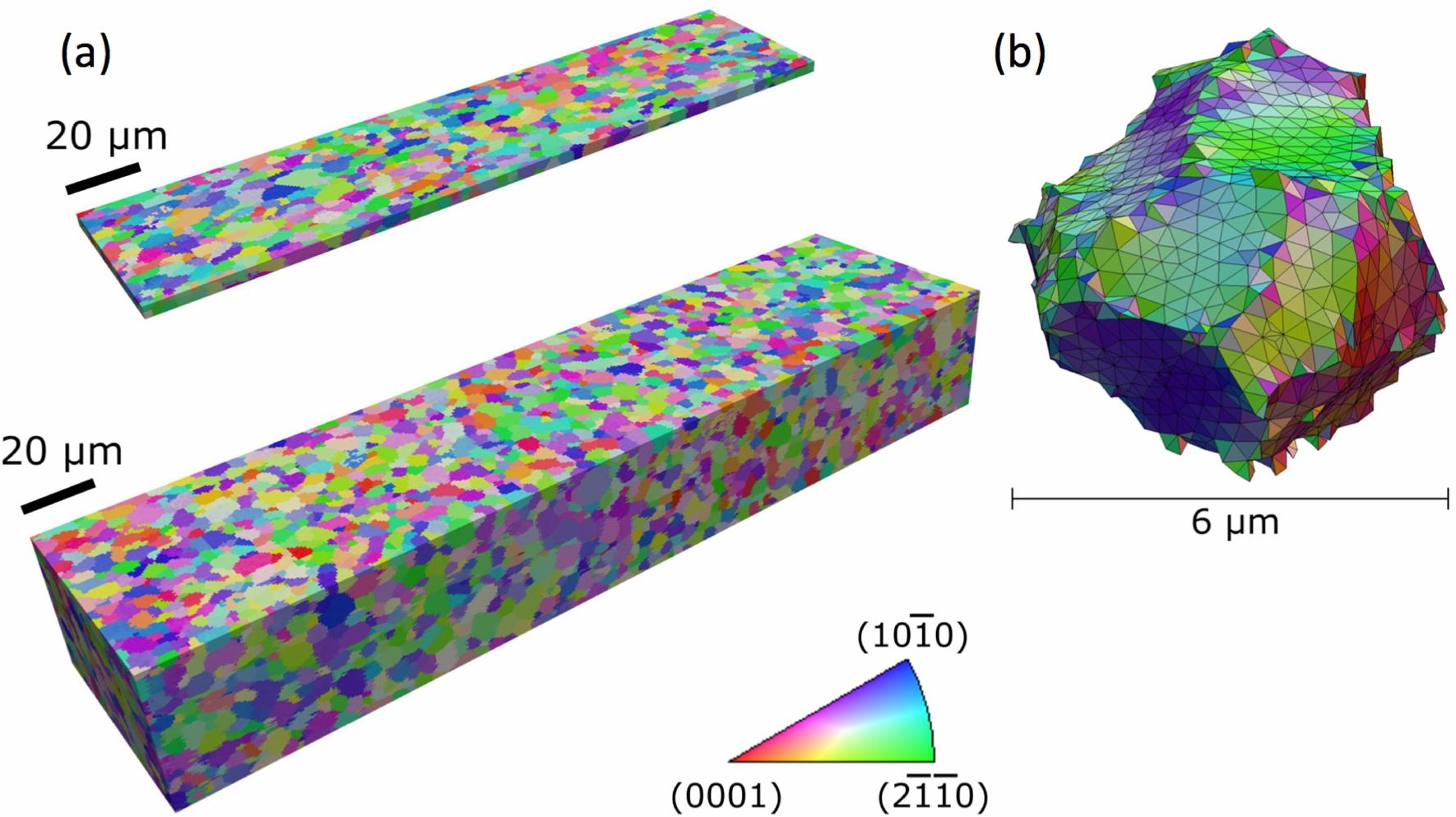


Figure 4

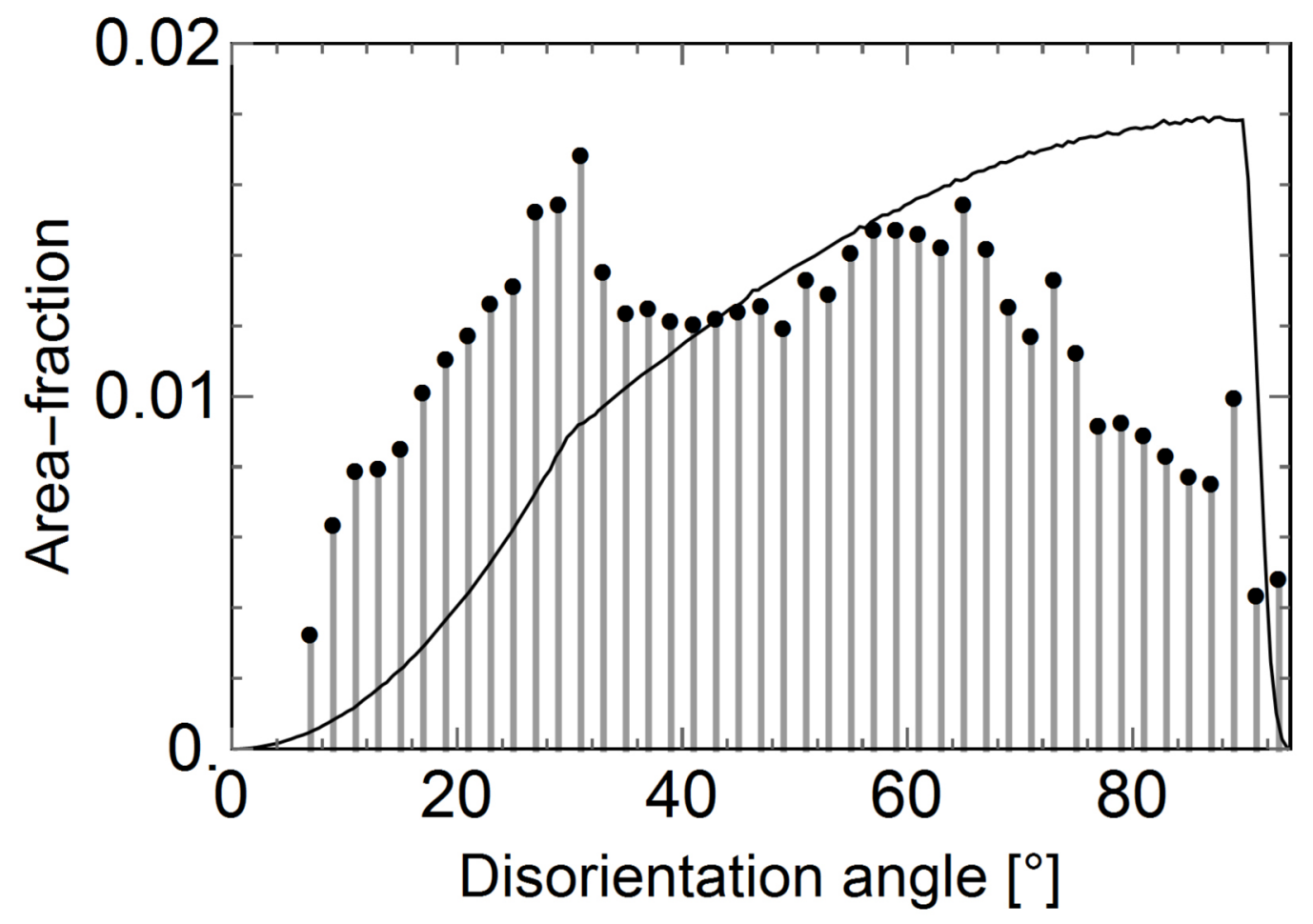




\section{Figure 5}

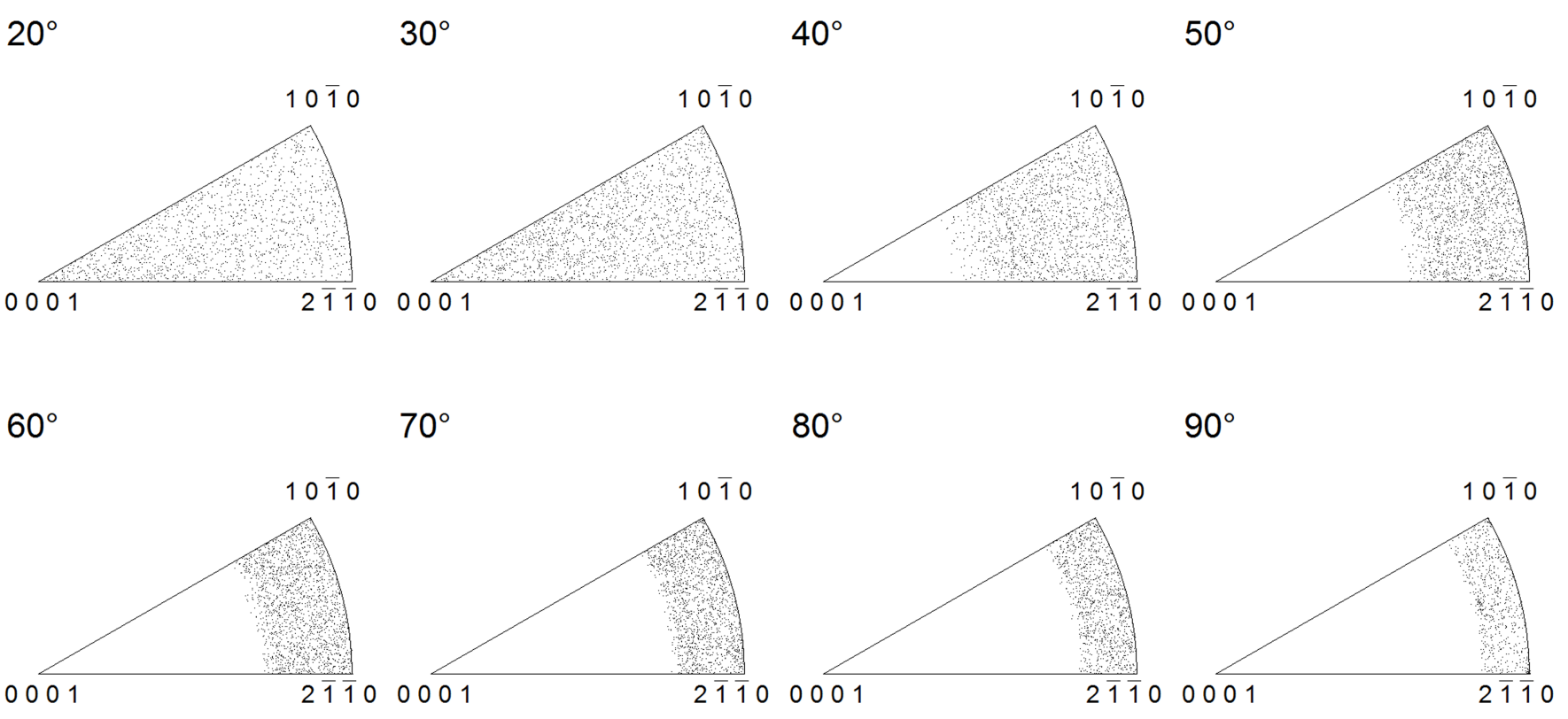


Figure 6

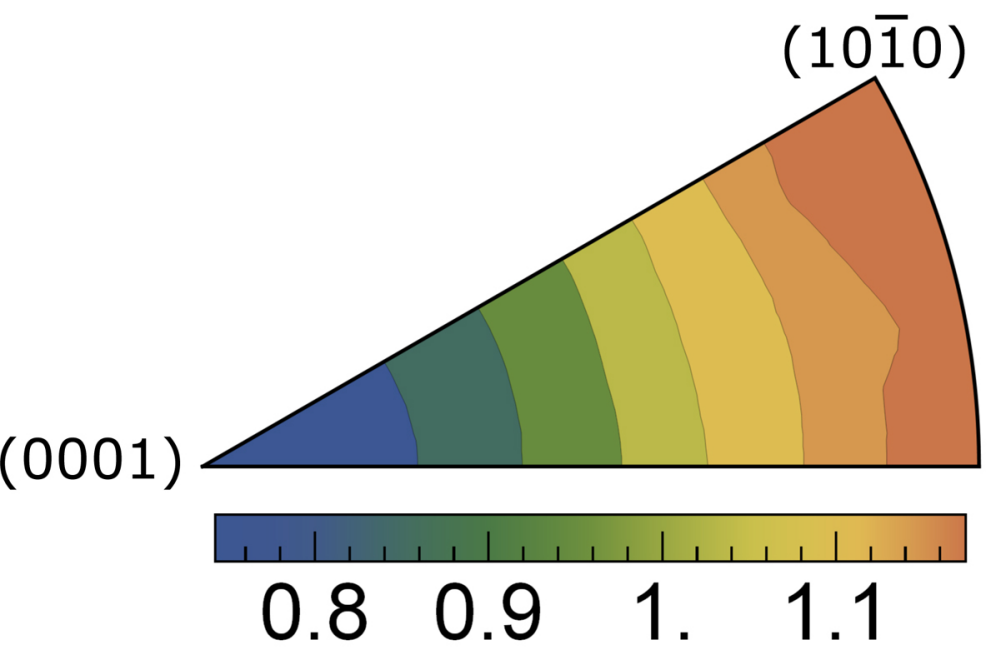

$(2 \overline{1} \overline{1} 0)$ 
Figure 7
(a) $22 \% /[0001]$
(b) $22^{\circ} /[10 \overline{1} 0]$
(c) $21^{\circ} /[2 \overline{1} \overline{1} 0]$
(d) $20^{\circ} /[4 \overline{1} \overline{3} 2]$

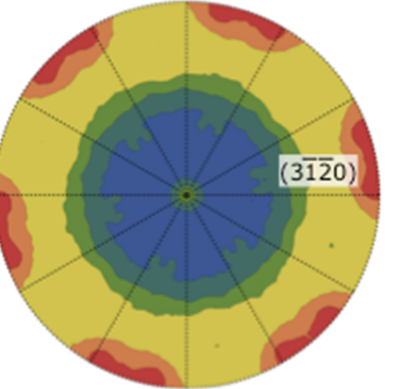

3. 4. 5. 6.7 .

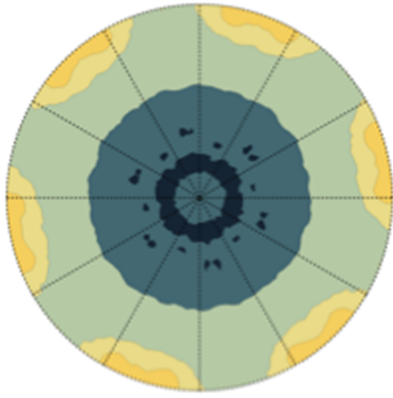

$\begin{array}{llll}0.5 & 0.6 & 0.7 & 0.8\end{array}$

(e) $28^{\circ} /[0001]$

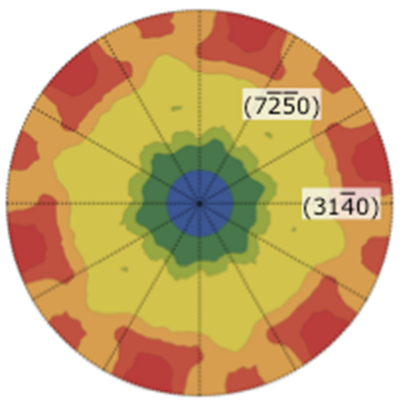

4.

6.

8.

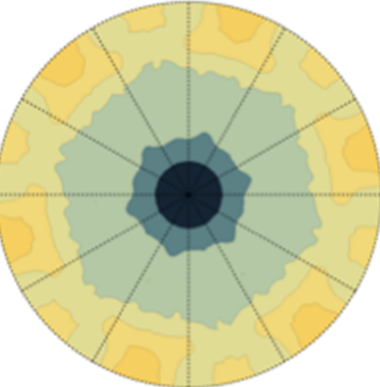

$\begin{array}{llll}0.5 & 0.6 & 0.7 & 0.8\end{array}$

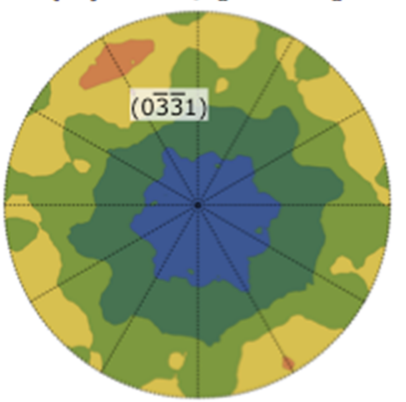

2. 3.4 . 4 .

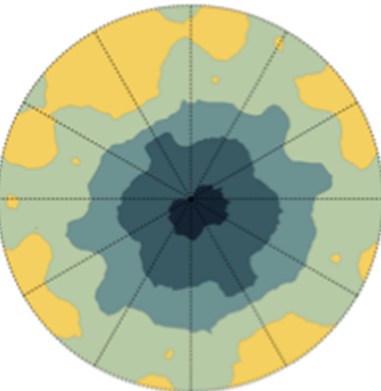

$\begin{array}{llll}0.4 & 0.5 & 0.6\end{array}$

(f) $90^{\circ} /[10 \overline{1} 0]$

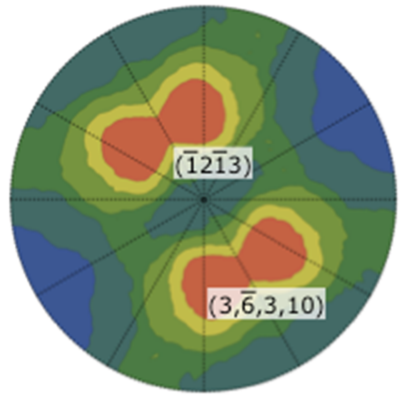

2. 3. 4.5 .

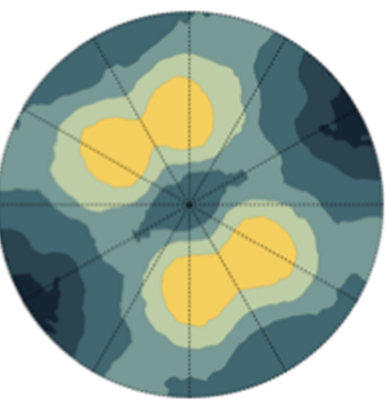

$0.5 \quad 0.6$
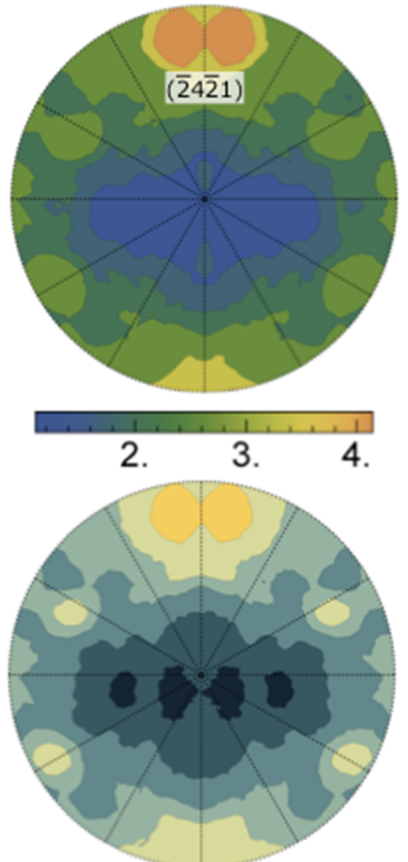

$\begin{array}{lll}0.4 & 0.5 & 0.6\end{array}$

(g) $31^{\circ} /[2 \overline{1} \overline{1} 0]$

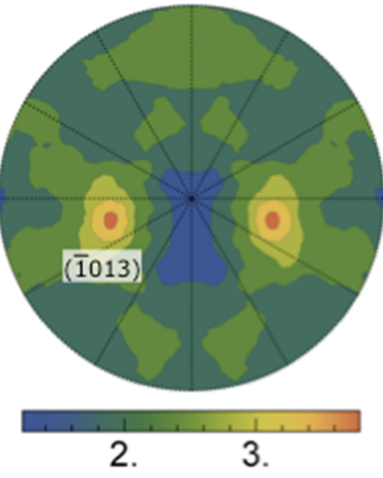

(h) $75^{\circ} /[2 \overline{1} \overline{1} 0]$

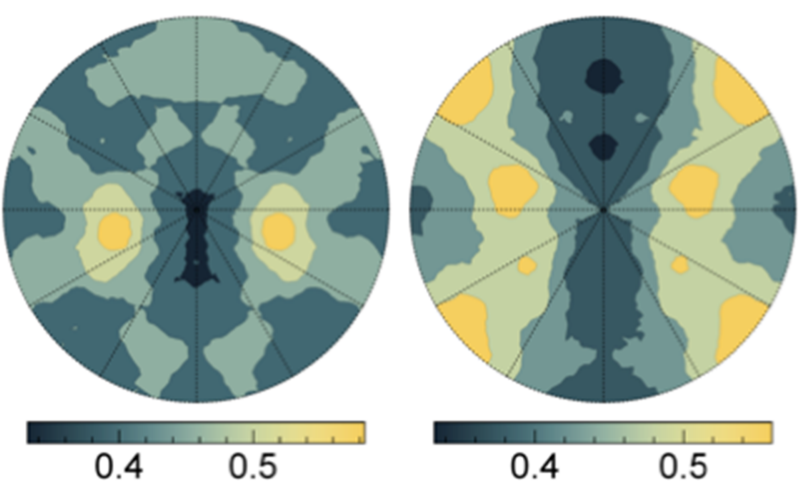




\section{Figure 8}

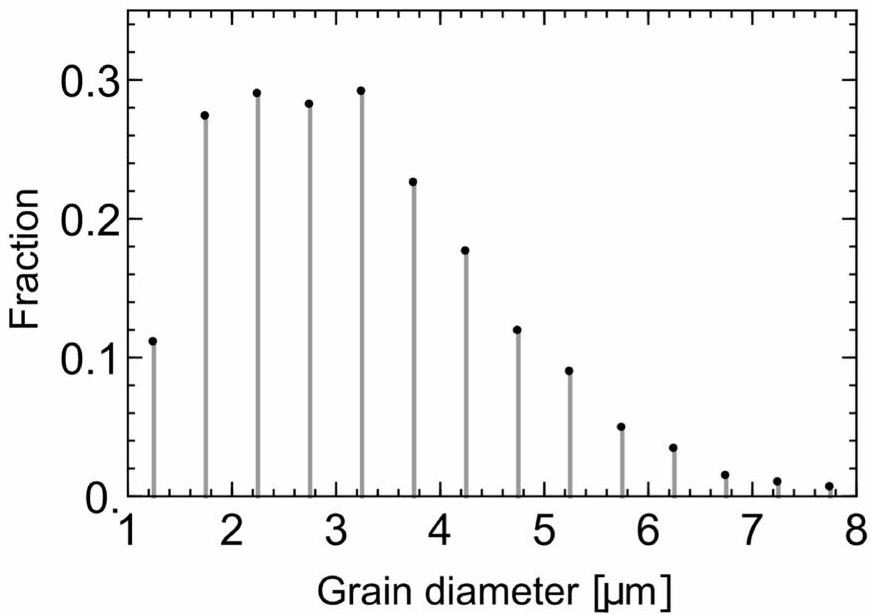


Figure 10

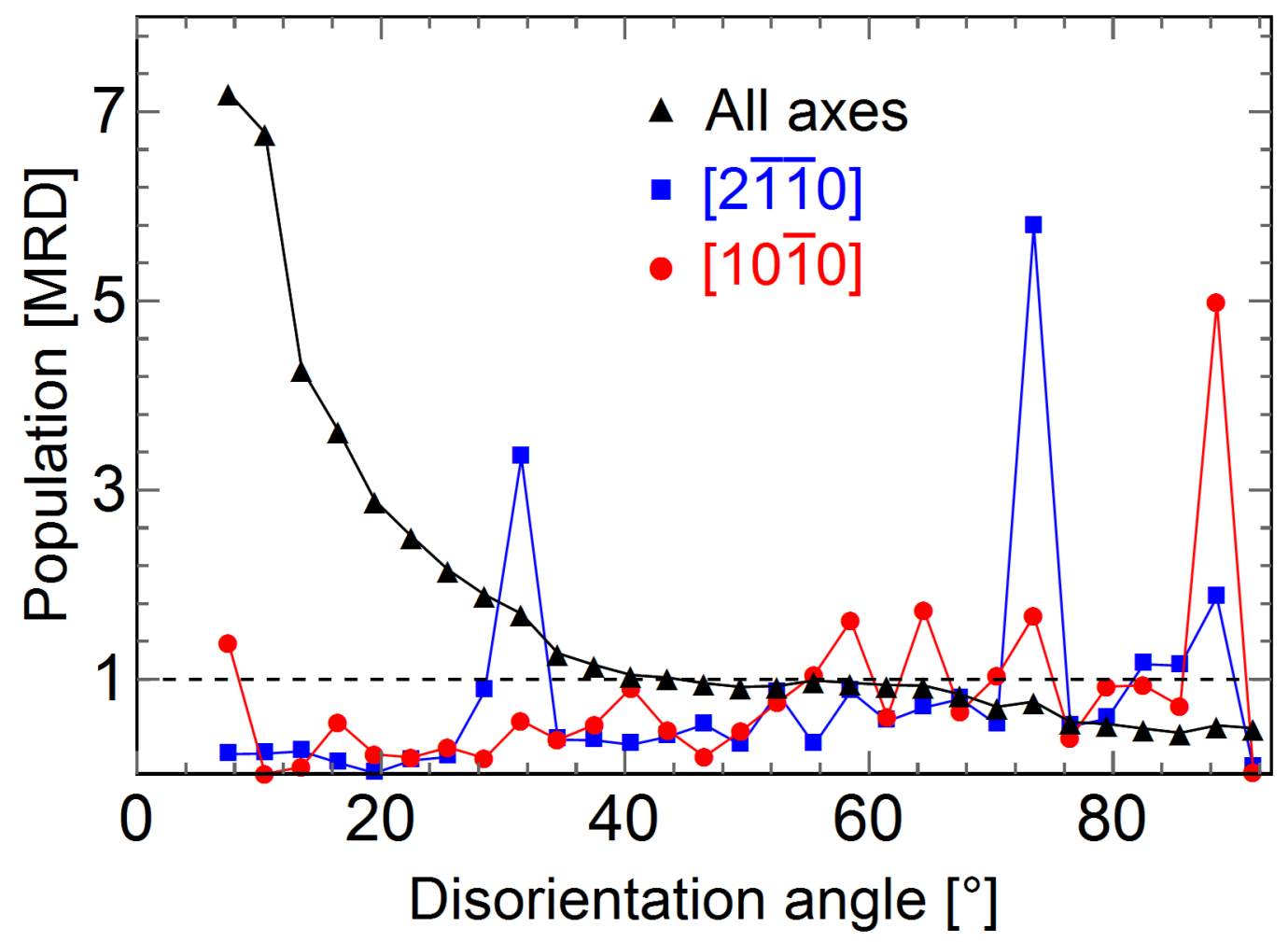




\section{Graphical abstract}
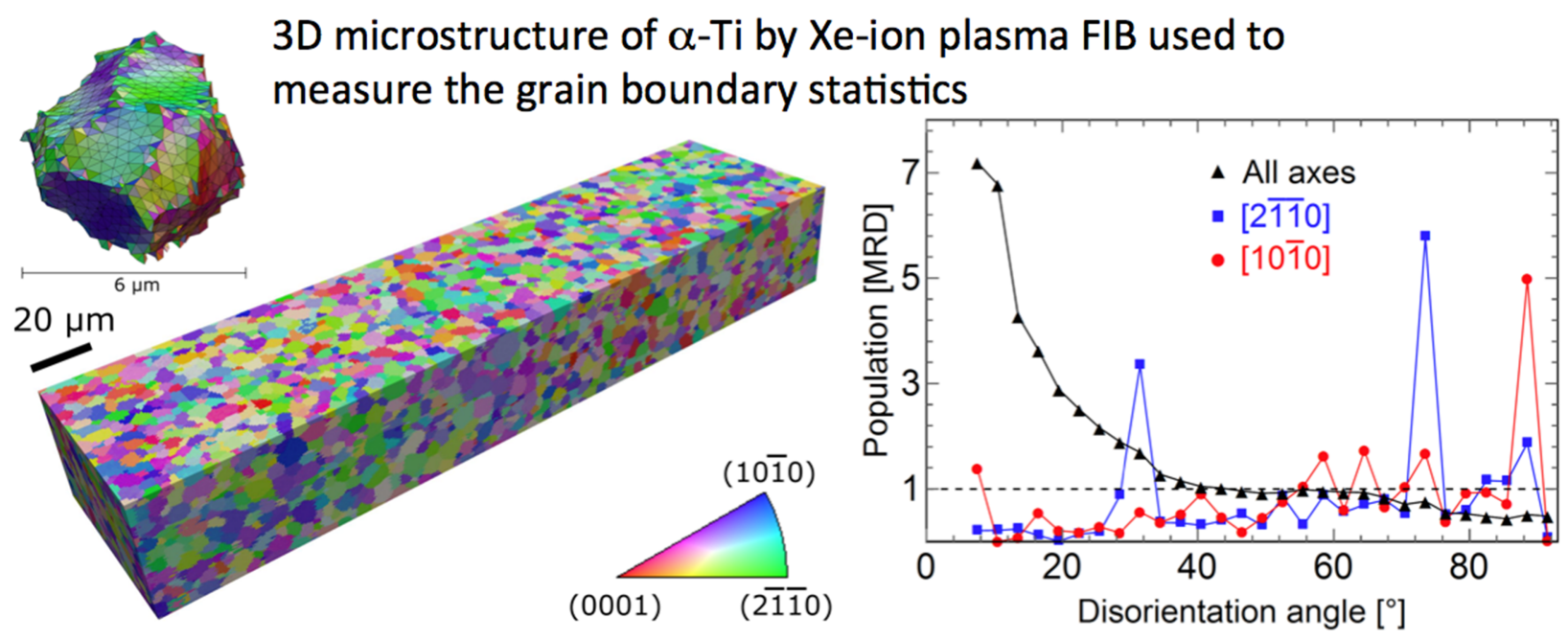